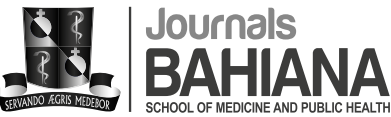

\section{Benefícios do flipped classroom no período pré-clínico: uma revisão sistemática}

\author{
Benefits of the flipped classroom in pre-clinical period: \\ a systematic review
}

\section{Ticiane Rodrigues Figueiredo', lêda Maria Barbosa Aleluia²}

1Autora para correspondência. Escola Bahiana de Medicina e Saúde Pública. Salvador, Bahia, Brasil. ORCID: 0000-0002-7451-8799. ticianerf@gmail.com 2Escola Bahiana de Medicina e Saúde Pública. Salvador, Bahia, Brasil. ORCID: 0000-0002-7979-1938. iedaaleulia@gmail.com

RESUMO | INTRODUÇÃO: Flipped Classroom (FC) é uma metodologia ativa de ensino na qual os níveis inferiores da taxonomia de Bloom são realizados em casa (estudando materiais disponibilizados por professores) e a sala de aula fica reservada para trabalhar os níveis superiores da taxonomia, através de discussões. OBJETIVO: Identificar os benefícios do método FC em comparação ao ensino tradicional nos anos pré-clínicos da graduação médica. MÉTODOS: Revisão sistemática através de pesquisa nos bancos de dados eletrônicos no período definido entre 31/12/1999 e 25/06/2017. RESULTADOS: Foram encontrados 262 artigos, dos quais 04 atenderam integralmente aos critérios de inclusão e exclusão previamente estabelecidos. O desempenho acadêmico mensurado através de provas aplicadas foi superior no método FC em dois estudos. Um estudo demonstrou que os alunos obtiveram melhor desempenho nas questões do tipo análise, segundo a taxonomia de Bloom. No método FC os alunos tiveram contato com mais fontes de estudo. Além disso, os estudos que trouxeram avaliação qualitativa dos discentes a respeito da metodologia obtiveram opiniões favoráveis à sua aplicação. CONCLUSÃO: $O$ método $\mathrm{FC}$ foi vantajoso quanto à motivação dos alunos. Os resultados encontrados sugerem que este método promova retenção maior do conteúdo. Tal metodologia se mostrou superior ao ensino tradicional nas questões do tipo análise, segundo a taxonomia de Bloom, indicando a possibilidade de que este método seja melhor para abordar níveis de complexidade maiores no processo de ensino-aprendizagem. Os dados relacionados ao desempenho acadêmico dos alunos em cada método ainda são inconclusivos.

PALAVRAS-CHAVE: Metodologia ativa. Ensino médico. Educação médica. Flipped Classroom.

\begin{abstract}
INTRODUCTION: Flipped Classroom (FC) is an active teaching methodology in which the lower levels of Bloom's taxonomy are realized at home: watching videos made available by teachers or studying through the teaching material offered, while the classroom is reserved to work the levels of taxonomy through discussions. OBJECTIVE: Identify the benefits of the HR method compared to traditional education in the pre-clinical years of medical graduation. METHODS: Systematic review through electronic database search in the period defined between 12/31/1999 and 6/25/2017. RESULTS: A total of 262 articles were found, of which 04 fully satisfy the inclusion and exclusion criteria previously established. The academic performance measured through applied tests was superior in the FC method in two studies. One study demonstrated that students performed better on analysistype questions, according to Bloom's taxonomy. In the FC method the students had contact with more sources of study. In addition, the studies that brought qualitative evaluation of the students about the methodology obtained opinions favorable to its application. CONCLUSION: The FC method was advantageous in motivating students. The results suggest that this method promotes greater content retention. The FC methodology becomes superior to traditional teaching in the analysis-type questions, according to Bloom's taxonomy, indicating the possibility that this method is better to address higher levels of complexity in the teaching-learning process. Data related to students' academic performance in each method are still inconclusive.
\end{abstract}

KEYWORDS: Active learning. Medical education. Flipped Classroom. Flip Classroom. Reverse classroom. 


\section{Introdução}

Em 1956, Benjamin S. Bloom estudou o processo de aprendizado e descreveu uma classificação para as suas etapas. Nasceu assim a Taxonomia de Bloom ${ }^{1-3}$ que vem sendo utilizada como ferramenta de comparação dos métodos de ensino. Seis etapas foram definidas hierarquicamente: conhecimento, compreensão, aplicação, análise, síntese e avaliação'. Para passar de uma etapa para outra é necessário o domínio da anterior ${ }^{1}$.

Novas maneiras de ensino surgiram e continuam sendo analisadas com auxílio da Taxonomia de Bloom. O flipped classroom (FC) é um modelo pedagógico de ensino ativo que teve seu nome cunhado em 2012 por Jonathan Bergmann e Aaron Sams ${ }^{4}$. Sua aplicação consiste em estudo de materiais em casa, incluindo visualização de vídeos ou leitura de textos recomendados pelos professores, enquanto a sala de aula é reservada à aplicação do conhecimento através de exercícios e discussões de casos com o auxílio e supervisão do docente ${ }^{5-11}$. Isto difere do método de ensino tradicional, no qual a etapa de "conhecimento" ocorre em sala de aula ${ }^{6}$. Na metodologia FC a sala de aula é utilizada para trabalhar os níveis mais avançados da taxonomia ${ }^{11}$.

Apenas a "inversão da sala de aula", no entanto, não contempla toda filosofia da metodologia FC: é necessário que o aprendizado que acontecia em grupo aconteça individualmente e o ambiente coletivo seja utilizado de forma dinâmica e interativa para aplicação do conteúdo aprendido ${ }^{12}$. Este modelo tem-se mostrado benéfico por diversas razões:

(a) quando um conceito errôneo está largamente difundido entre os alunos, professores tem oportunidade de identificar falhas na transmissão de conceitos através da realização da atividade ${ }^{10}$;

(b) estudantes podem aprender no seu próprio ritmo, pausando ou repetindo o vídeo quantas vezes desejarem ${ }^{6,8,13}$;

(c) atividades em sala de aula permitem que professores percebam as dificuldades de seus alunos e atuem em cima destas ${ }^{6,13}$;

(d) vídeos sempre disponíveis para o acesso permitem que os estudantes ausentes possam ter acesso ao material ${ }^{13}$; (e) especialistas que não poderiam comparecer à sala de aula podem gravar suas aulas e enviar aos alunos;

(f) acesso a aulas do mesmo tema por diversos professores, promovendo percepção de outros ângulos, facilitando o aprendizado ${ }^{13}$;

(g) professores podem melhorar seu desempenho profissional assistindo aulas de outros professores ${ }^{13}$.

Apesar de ser um método bastante interessante, existem diversos fatores que podem convergir para falha da aplicação. O funcionamento efetivo requer esforço por parte da faculdade e dos alunos. Estes precisam se preparar para participar ativamente da discussão. Isto requer conhecimento prévio do assunto e tempo livre, além de orientação quanto aos objetivos para que se sintam motivados ${ }^{10}$.

Na literatura, a eficiência do método FC no curso de medicina é avaliada sem distinção entre os períodos da graduação. No entanto, a abordagem e objetivo de ensino da etapa "pré-clínica" (até o $8^{\circ}$ semestre) é diferente do internato e da residência. Portanto, são necessárias revisões que avaliem cada grupo independentemente, respeitando suas particularidades ${ }^{14}$. Dentro deste contexto, a presente revisão busca avaliar se há benefícios no uso deste método na etapa "pré-clínica" do curso de medicina em comparação com o modelo tradicional de ensino.

\section{Objetivo}

Identificar os benefícios do método flipped classroom em comparação ao ensino tradicional nos anos préclínicos da graduação médica.

\section{Método}

Uma revisão sistemática foi realizada utilizando os bancos de dados PubMed (Public Medical Literature Analysis and Retrieval System Online), LILACS (Literatura Latino-Americana e do Caribe em Ciências da Saúde) e Scielo (Scientific Electronic Library Online). Foram utilizados os seguintes descritores: "(Flipped) AND classroom”; “(Flip) AND classroom”; “(reverse) AND classroom". O período da busca dos artigos foi definido entre 31/12/1999 e 25/06/2017. Foram 
incluídos artigos de língua inglesa, portuguesa ou espanhola que avaliaram o uso do modelo flipped classroom no ensino médico durante primeiro ao quarto ano da graduação de medicina. Os seguintes critérios de exclusão foram utilizados: artigos que não compararam o método flipped classroom com o método tradicional, abordaram sobre medicina chinesa, não utilizaram em sua metodologia apenas estudantes de medicina, não explicitaram em sua metodologia qual curso e período cursado pelos estudantes, estudos feitos com internos ou residentes, anais de congresso, relatório de simpósios, jornadas, congressos ou resumos de artigos.

\section{Resultados}

\section{a) Identificação e seleção dos estudos}

Foram encontrados 262 artigos na pesquisa em banco de dados, 177 artigos foram excluídos por análise do título, restando 85 para serem avaliados na próxima etapa. A partir da análise do resumo 36 artigos foram excluídos, restando 49 para análise da metodologia. Para etapa de leitura integral foram selecionados seis artigos, dos quais dois foram excluídos por não abordarem a comparação entre o método tradicional e o flipped classroom. Os motivos de exclusão de cada artigo estão explicitados no fluxograma 1.

Fluxograma 1. Processo de seleção de artigos

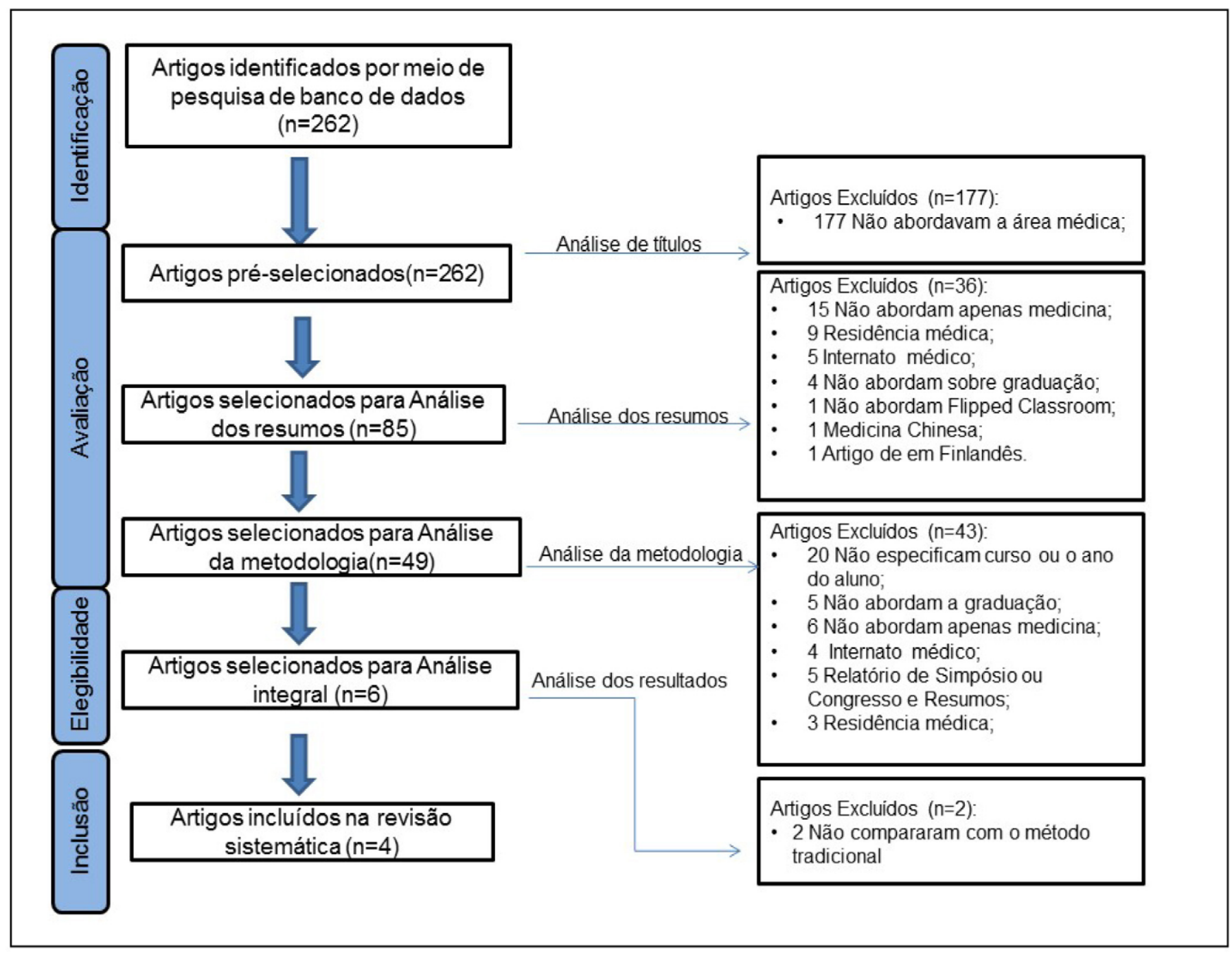

Fonte: As autoras (2019). 


\section{b) Análise da qualidade dos artigos}

O Strengthening the Reporting of Observational Studies in Epidemiology (STROBE) indica 22 critérios que deveriam ser atendidos em estudos observacionais. Para o cumprimento integral de cada critério foi atribuído 1 ponto; cumprimento parcial 0,5 ponto e o não cumprimento 0 ponto. A pontuação média dos artigos selecionados foi 17,87, representando um cumprimento de $81,22 \%$ dos critérios pré-estabelecidos.

Quadro 1. Avaliação da qualidade dos estudos selecionados

\begin{tabular}{|c|c|c|c|c|}
\hline TÓPICO & Veeraman et al. ${ }^{(4)}$ & Sajid et al. ${ }^{(9)}$ & Morton et al. ${ }^{(15)}$ & Zeng et al. ${ }^{(8)}$ \\
\hline \multicolumn{5}{|l|}{ Título e Resumo } \\
\hline \multicolumn{5}{|l|}{ Introdução } \\
\hline \multicolumn{5}{|l|}{ Conteúdo/Justificativa } \\
\hline \multicolumn{5}{|l|}{ Objetivos } \\
\hline \multicolumn{5}{|l|}{ Métodos } \\
\hline \multicolumn{5}{|l|}{ Desenho do estudo } \\
\hline \multicolumn{5}{|l|}{ Contexto } \\
\hline \multicolumn{5}{|l|}{ Participantes } \\
\hline \multicolumn{5}{|l|}{ Variáveis } \\
\hline \multicolumn{5}{|l|}{ Fontes de dados/Mensuração } \\
\hline \multicolumn{5}{|l|}{ Viés } \\
\hline \multicolumn{5}{|l|}{ Tamanho do estudo } \\
\hline \multicolumn{5}{|l|}{ Variáveis quantitativas } \\
\hline \multicolumn{5}{|l|}{ Métodos estatísticos } \\
\hline \multicolumn{5}{|l|}{ Resultados } \\
\hline \multicolumn{5}{|l|}{ Participantes } \\
\hline \multicolumn{5}{|l|}{ Dados descritivos } \\
\hline \multicolumn{5}{|l|}{ Desfecho } \\
\hline \multicolumn{5}{|l|}{ Resultados principais } \\
\hline \multicolumn{5}{|l|}{ Outras análises } \\
\hline \multicolumn{5}{|l|}{ Discussão } \\
\hline \multicolumn{5}{|l|}{ Interpretação } \\
\hline \multicolumn{5}{|l|}{ Generalização } \\
\hline \multicolumn{5}{|l|}{ Outras informações } \\
\hline \multicolumn{5}{|l|}{ Financiamento } \\
\hline TOTAL & 15,5 & 18 & 21 & 16 \\
\hline
\end{tabular}

\section{c) Características gerais dos estudos selecionados}

As características gerais e a forma como os artigos incluídos aplicaram a metodologia FC estão descritas na tabela 1. 
Tabela 1. Características gerais dos estudos selecionados

\begin{tabular}{|c|c|c|c|c|c|}
\hline Referência & País e Ano & $\mathrm{n}$ & $\begin{array}{l}\text { Ano em curso } \\
\text { do estudante }\end{array}$ & Duração & Intervenção \\
\hline $\begin{array}{l}\text { Veeraman et } \\
\text { al. }^{(4)}\end{array}$ & Índia, 2015 & 130 & $1^{\circ}$ ano & 2 semanas & $\begin{array}{l}\text { Os estudantes foram divididos em } 5 \text { grupos de } 26 \\
\text { pessoas. Cada grupo recebeu } 5 \text { casos clínicos e } 3 \\
\text { perguntas que orientavam o objetivo de cada caso. } \\
\text { Após a leitura do material fornecido, ocorreu discussão } \\
\text { em grupo com a presença de facilitador. }\end{array}$ \\
\hline Sajid et al. ${ }^{(9)}$ & $\begin{array}{l}\text { Arábia } \\
\text { Saudita, } \\
2016\end{array}$ & 154 & $3^{\circ}$ ano & 6 meses & $\begin{array}{l}\text { As aulas foram colocadas no ambiente virtual com } \\
\text { áudio contendo orientações anexadas. Foram criados } \\
\text { fóruns de discussão online onde os alunos poderiam } \\
\text { tirar suas dúvidas com os professores. }\end{array}$ \\
\hline Morton et al. ${ }^{(15)}$ & $\begin{array}{l}\text { Estados } \\
\text { Unidos, } \\
2016\end{array}$ & 203 & $1^{\circ}$ ano & 17 semanas & $\begin{array}{l}\text { Disponibilizou-se } 30 \text { horas de vídeo dos assuntos do } \\
\text { semestre. Em anexo, recebiam também orientações e } \\
\text { banco de imagens de anatomia. A discussão em classe } \\
\text { ocorria no modelo "think-pair-share" e, neste momento, } \\
\text { os assuntos eram complementados e erros corrigidos } \\
\text { pelo orientador. }\end{array}$ \\
\hline Zeng et al. ${ }^{(8)}$ & China, 2017 & 181 & Ciclo básico & 3 semanas & $\begin{array}{l}\text { Foram divididos randomicamente em dois grupos: } 90 \\
\text { para metodologia FC e } 91 \text { no grupo controle. Alunos do } \\
\text { método FC recebiam vídeo-aulas e exercícios; } \\
\text { realizavam discussões em sala de aula. Os dois grupos } \\
\text { receberam o mesmo livro texto, mesmos professores e } \\
\text { formato de prova. }\end{array}$ \\
\hline
\end{tabular}

FC: Flipped Classroom.

\section{d) Eficácia do flipped classroom}

No artigo produzido por Veeraman et al. ${ }^{4}$ foi aplicado um pré-teste (antes do início do módulo) e um pós-teste (ao final do módulo). Após uma semana, realizaram outra prova com o conteúdo trabalhado e a performance foi comparada ao pós-teste. Os alunos que passaram pelo método FC obtiveram nota melhor nesta comparação do que os alunos do mesmo módulo pelo ensino tradicional. O desempenho no pós-teste foi consideravelmente melhor do que o pré-teste (dados na tabela 2 ), com $p<0,05$. Foi observada uma correlação positiva $(0,5)$ entre o pós-teste e a prova realizada posteriormente.

No mesmo estudo, a percepção dos discentes a respeito do modelo de ensino foi avaliada através de dois questionários: um com questões fechadas avaliando o método em 1-5 (entre ruim e muito bom) e outro aberto com questões que induziam os estudantes a fazerem comentários qualitativos do método. $86 \%$ dos alunos sentiram que o método FC foi mais efetivo em cumprir os objetivos de aprendizagem do que o método tradicional. $78 \%$ dos alunos sentiram-se fortemente encorajados a se engajar no aprendizado antes da aula.

No estudo descritivo analítico conduzido por Sajid et al. ${ }^{9}$ utilizou-se como método avaliativo uma prova online com questões de múltipla escolha que abrangia os assuntos dados através do método FC e tradicional, comparando seu desempenho. A performance destes estudantes foi comparada também com a dos alunos no ano anterior (2014), no qual foi aplicado apenas a metodologia tradicional. A opinião dos alunos a respeito da metodologia foi coletada através de um questionário fechado (respostas entre 1-5). 73.1\% dos estudantes preferiram a leitura online, $69 \%$ ficou satisfeito com a abordagem, $81 \%$ considerou a metodologia FC melhor do que a tradicional. A turma de 
2014 possuía 155 estudantes, enquanto a de 2016, 155 estudantes ( $t=0,142$ e $p=0,887)$. As notas " $A$ " e " $B$ " foram mais frequentes e as notas " $\mathrm{C}$ " $\mathrm{e}$ " $\mathrm{F}$ " menos frequentes em 2015, quando comparado a 2014, mas sem significância estatística $\left(t_{(307)}=0,142, p=0,887\right)$. $O$ desempenho dos alunos no método tradicional foi semelhante ao método FC, conforme pode ser observado na tabela 2 e no gráfico 1.

O estudo realizado por Morton et al..$^{15}$ foi feito em duas turmas em dois anos consecutivos. Em 2013 a turma foi submetida ao método tradicional combinado com uma metodologia ativa: o think-pair-share, na qual os alunos recebiam problemas, pensavam na solução sozinhos, depois discutiam com seus pares. Em um segundo momento um grupo era aleatoriamente escolhido para compartilhar as respostas com a turma. A turma de 2014 foi submetida a metodologia FC. Foram disponibilizados vídeo-aulas e aulas em classe, onde ocorria também metodologia think-pair-share. O desempenho acadêmico dos alunos pode ser visto na tabela 2 . As questões da prova foram classificadas segundo a taxonomia de Bloom ${ }^{1-3}$. Os alunos no método FC obtiveram melhor desfecho nas questões do tipo "análise". O desempenho dos alunos em cada tipo de questão foi:

a. Compreensão e reconhecimento: Tradicional $90 \pm 8 \%$ e FC $88 \pm 7 \%$;

b. Aplicação: Tradicional $88 \pm 15 \%$ e FC $87 \pm 16 \%$

c. Análise: Tradicional $92 \pm 13 \%$ e FC $93 \pm 13 \%$.

Após a análise de análise de amostras não paramétricas pelo "The Mann Whitney test" foi observado que não havia diferença nas questões do tipo compreensão e reconhecimento; a diferença entre as questões do tipo aplicação não era estatisticamente significante (método FC $1 \%$ superior), mas nas questões do tipo análise a diferença foi relevante (método FC 3\% superior).

O estudo realizado por Zeng et al. ${ }^{8}$ utilizou dois grupos divididos aleatoriamente. Uma semana após cada assunto os alunos dos dois grupos eram submetidos a uma prova. O grupo FC obteve pontuação maior do que o grupo tradicional, conforme pode ser observado na tabela 2. Os alunos do grupo FC receberam um questionário para avaliar de 1 a 5 a metodologia utilizada. A maioria dos alunos avaliou positivamente a metodologia FC quanto ao estímulo do estudo individual, mas afirmaram que os assuntos são ensinados de forma fragmentada e não sistemática, além disso aumentou sua carga de estudos. Os alunos no método FC gastaram mais tempo se preparando para a aula do que o do grupo controle ( $42 \pm 22 \mathrm{~min}$ vs $30 \pm 10 \mathrm{~min})$, no entanto, não houve diferença significativa no tempo gasto depois da aula entre o grupo FC $(56 \pm 46 \mathrm{~min})$ e o tradicional ( $54 \mathrm{~min} \pm 31 \mathrm{~min})$. Os professores foram entrevistados quanto a sua percepção do método e ao tempo extraclasse que a atividade demandou. Os professores gastaram em média 30 horas para se preparar para a fase em sala de aula e 6 horas para preparação dos vídeos, enquanto no método tradicional dispenderam apenas 10 horas.

O gráfico 1 demonstra o desempenho dos estudantes no método tradicional e FC. Este desempenho foi mensurado através da aplicação de testes nos dois grupos, conforme a metodologia das avaliações é explicada na tabela 2. Os artigos demonstraram uma pequena diferença no desempenho dos alunos, com exceção do estudo de Veeraman et al. ${ }^{4}$ que demonstrou desempenho consideravelmente maior no método FC.

Gráfico 1. Impacto do método flipped classroom no aprendizado

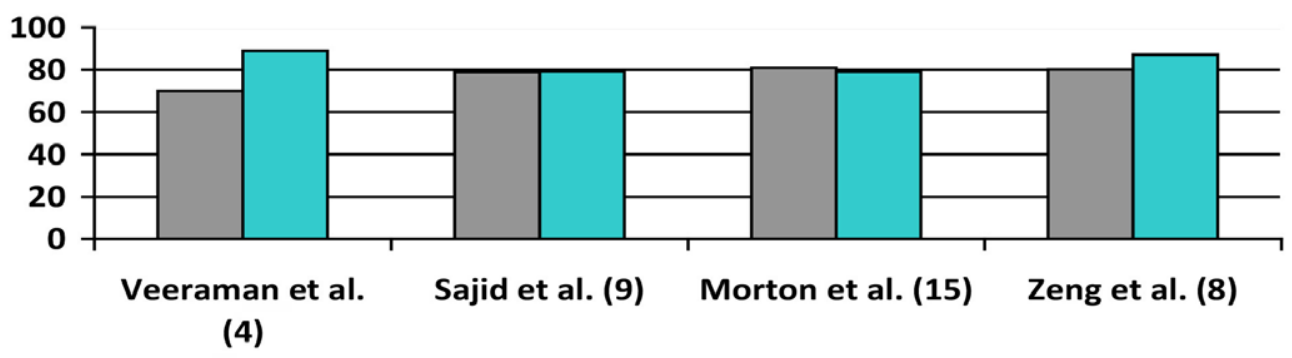

$\square$ Tradicional $\square$ Flipped Classroom

Fonte: As autoras (2019). 


\begin{tabular}{|c|c|c|c|}
\hline Referência & Metodologia Flipped Classroom & Método de Avaliação & Resultado \\
\hline $\begin{array}{l}\text { Veeraman et } \\
\text { al. }^{(4)}\end{array}$ & $\begin{array}{l}5 \text { casos clínico foram disponibilizados com os } \\
\text { objetivos de aprendizagem e textos. Em sala } \\
\text { ocorria pré-teste e depois um facilitador escolhia } \\
\text { um aluno para responder cada questão, } \\
\text { compartilhando a discussão com a turma e } \\
\text { suplementando a informação. }\end{array}$ & $\begin{array}{l}\text { Pré-teste e pós-teste em classe e depois } \\
\text { de uma semana foi aplicada uma prova. }\end{array}$ & $\begin{array}{l}\text { Tradicional: } 70 \pm 14 \% \\
\text { Pré-teste: } 3,35 \pm 1,5 ; \\
\text { Pós-teste: } 8,12 \pm 1,6 ; \\
\text { Prova após pós-teste: } 89 \pm 12 \%\end{array}$ \\
\hline Sajid et al. ${ }^{(9)}$ & $\begin{array}{l}\text { Vídeo-aulas foram disponibilizadas junto a um } \\
\text { fórum online, onde era possivel tirar as dúvidas } \\
\text { com professores. }\end{array}$ & $\begin{array}{l}\text { Prova de múltipla escolha aplicada } \\
\text { online. }\end{array}$ & $\begin{array}{l}\text { Tradicional: } 78,81 \\
\text { FC: } 79,05\end{array}$ \\
\hline Morton et al. ${ }^{(15)}$ & $\begin{array}{l}\text { O grupo tradicional recebia também a } \\
\text { metodologia ativa através do think-pair-share. O } \\
\text { grupo FC recebia vídeos aulas que continham em } \\
\text { anexo objetivos de aprendizagem, texto e } \\
\text { animações. Em sala de aula ocorria a dinâmica } \\
\text { "think-pair-share". }\end{array}$ & $\begin{array}{l}\text { Os dois grupos responderam uma prova } \\
\text { com } 150 \text { questões de múltipla escolha. }\end{array}$ & $\begin{array}{l}\text { Tradicional: } 81 \pm 8 \% \\
\text { FC: } 79 \pm 7 \%\end{array}$ \\
\hline Zeng et al. ${ }^{(8)}$ & $\begin{array}{l}\text { Estudo randomizado com dois grupos: tradicional } \\
\text { e FC. Os dois grupos possuíam os mesmos } \\
\text { professores. O grupo FC recebia vídeo-aulas e } \\
\text { exercícios. Em sala eram tiradas as dúvidas, } \\
\text { realizado discussões, exercícios e o professor } \\
\text { suplementava a informação. }\end{array}$ & $\begin{array}{l}\text { Uma semana após o modulo foi } \\
\text { aplicada uma prova para os dois grupos. }\end{array}$ & $\begin{array}{l}\text { Tradicional: } 8,03 \pm 1,01 \\
\text { FC: } 8,72 \pm 1,01\end{array}$ \\
\hline
\end{tabular}

\section{Discussão}

O desempenho acadêmico mensurado através das provas aplicadas foi superior na metodologia FC, quando comparado ao método tradicional em dois estudos ${ }^{4,8}$. Porém, no estudo de Morton et al. ${ }^{15}$ o método FC obteve resultado inferior ao tradicional. Este artigo, no entanto, utilizou uma metodologia ativa dentro do que o autor chamou de "método tradicional": o "think-pair-share". Isto é um viés a ser considerado, pois a presença do "think-pair-share" no grupo tradicional pode ter mascarado os benefícios do FC: uma vez que ambas são metodologias ativas, a inserção do "think-pair share" pode ter ofuscado benefícios do FC. Esta hipótese aponta que um estudo para comparar a eficácia dos métodos de ensino ativos entre si pode caracterizar se existe benefício adicional com o uso do FC perante os outros modelos ativos.
Além de comparar o desempenho entre as metodologias "tradicional" e FC de maneira global, Morton et al. ${ }^{15}$ encontraram que o método FC foi superior ao grupo tradicional especialmente naquelas questões do tipo análise, segundo a taxonomia de Bloom ${ }^{1-3}$. Outros estudos na literatura corroboram esse achado, uma vez que por se tratar de uma metodologia ativa, centrada no estudante, promove maior reflexão e análise crítica dos problemas apresentados $^{7,11,16}$. Uma meta-análise feita com 225 artigos comparando a eficácia entre o método tradicional e as metodologias ativas encontrou que os alunos possuem rendimento superior em $6 \%$ quando inseridos na abordagem ativa, enquanto aqueles submetidos ao método tradicional possuem 1,5 vezes mais chance de falhar no desempenho mínimo estabelecido pela universidade ${ }^{17}$. 
Também a respeito da taxonomia de Bloom ${ }^{1-3}$, sabe-se que os estudantes necessitam de mais auxílio durante a aprendizagem dos níveis de maior complexidade quando comparado aos níveis inferiores (conhecimento e compreensão) ${ }^{5}$. O método FC torna-se efetivo neste contexto já que trabalha os níveis mais elevados (aplicação, análise e síntese) em sala de aula. Portanto, pode-se atribuir o melhor desempenho dos alunos nas questões do tipo análise ao fato desta metodologia trabalhar a capacidade de extrapolação do conteúdo aprendido e sua aplicação em situações diversas em sala de aula.

Veeraman et al. ${ }^{4}$ obtiveram resultado positivo quanto ao desempenho dos alunos no método FC com grande diferença percentual do método tradicional. Esse desfecho pode ter sido secundário à diferença em sua metodologia do estudo, quando comparado aos outros trabalhos. O artigo comparou o desempenho dos alunos aplicando a prova uma semana após o fim do módulo, enquanto os outros estudos fizeram a comparação utilizando apenas a prova ao fim do módulo. Tal resultado levanta a hipótese de que a metodologia FC promova melhor retenção do conteúdo aprendido a longo prazo.

Todos artigos que trouxeram dados sobre a análise qualitativa dos discentes quanto ao método FC obtiveram resultados favoráveis ${ }^{4,8,9}$. Os alunos sentiramse mais encorajados a estudar e acharam a metodologia FC mais eficiente do que o método tradicional. A literatura mostra que, em geral, os alunos aprovam tal abordagem visto que o ensino através de vídeos curtos permite a manutenção da atenção no assunto, facilitando a compreensão. $O$ assunto é passado de maneira mais direta, evitando que informações desnecessárias prolonguem a aula, como geralmente ocorre no método tradicional ${ }^{18}$. Outros artigos corroboram com essas informações evidenciando que o FC promoveu maior engajamento dos alunos no processo de aprendizagem, encorajando-os a serem responsáveis pelo seu próprio aprendizado $0^{5,7,18}$.

Alguns discentes, entretanto, relatam que o método FC seria adequado apenas para conceitos básicos ou que já foram estudados em outro momento e que a redução do contato com o professor atua como empecilho para o aprendizado 9 . Os dados encontrados na literatura estão em desacordo com tal informação trazendo que, apesar da redução do tempo de contato presencial entre discentes e docentes, a qualidade da interação é superior, de modo que os alunos se sentem satisfeitos com a efetividade do contato presencial ${ }^{7,18}$. Outros alunos comentaram que a forma que o método FC foi aplicada era fragmentada e desorganizada ${ }^{8}$. Isto mostra a relevância, já observada na literatura ${ }^{7,19}$, de explicar como a metodologia será aplicada. Importante também deixar claro os objetivos pedagógicos para fazer com que se sintam mais engajados, evitando a sensação de estarem "perdidos". O ensino através de uma metodologia ativa facilita o engajamento dos alunos, fazendo com que eles compreendam "o quê" e o "porquê" estão aprendendo aquilo ${ }^{16}$. É preciso também ajustar as provas aplicadas, de modo que sejam feitas perguntas que avaliem os níveis mais altos da taxonomia de Bloom ${ }^{1-3,7}$. Caso a prova permaneça focando nos níveis mais baixos da taxonomia, os alunos ficarão insatisfeitos, já que o foco principal do ensino foi deslocado a avaliação precisa ser coerente com tal modificação.

O trabalho escrito por Zeng et al. ${ }^{8}$ trouxe dados interessantes a respeito da qualidade do estudo e do tempo gasto por discentes e docentes no método FC. Os alunos submetidos a este método utilizaram mais tempo se preparando para a aula do que o os alunos no método tradicional, no entanto, não houve diferença significativa no tempo gasto depois da aula entre o grupo FC e o tradicional. Aqueles submetidos a metodologia FC, entretanto, tiveram contato com mais fontes de informação, enquanto o outro grupo revisava suas anotações ou a aula disponibilizada, o grupo FC variava suas fontes de estudo. Os professores, por sua vez, utilizaram em média 30h para se preparar para a fase em sala de aula e 6 horas para preparação dos vídeos, enquanto no método tradicional dispenderam apenas $10 \mathrm{~h}$. Apesar desta diferença, consideraram a atmosfera mais agradável na metodologia FC, com os alunos mais interessados, o que promovia um estímulo adicional. $\mathrm{O}$ aumento do tempo utilizado pelos professores na preparação do material em ambiente online já foi retratada na literatura ${ }^{5,7}$. O entusiasmo do facilitador também é um aspecto relevante para a qualidade do ensino e engajamento do aluno, repercutindo, portanto, na eficácia da implantação desta metodologia ${ }^{7}$. Neste contexto, é importante que exista uma avaliação qualitativa da metodologia adotada, que permita a emissão das opiniões dos discentes acerca da metodologia implantada7,16. Através das opiniões obtidas, os ajustes poderão ser realizados, feedbacks repassados aos professores, afim de que possam melhorar sua abordagem e se engajar com tal metodologia, pois só assim serão capazes de promover tal engajamento nos alunos. 


\section{Conclusão}

Os achados obtidos nessa revisão sugerem que o método FC promove uma retenção maior do conteúdo. A metodologia FC se mostrou superior ao ensino tradicional nas questões do tipo análise, segundo a taxonomia de Bloom ${ }^{1-3}$, indicando a possibilidade de que este método seja melhor para abordar níveis de complexidade maiores no processo de ensino-aprendizagem. O método flipped classroom foi vantajoso quanto a motivação dos alunos para estudar e, com discentes empenhados, os professores se sentem estimulados a lecionar. Os discentes, por sua vez, dedicaram mais tempo estudando antes das aulas e acessaram mais fontes de estudo, quando comparados àqueles no método tradicional. Os dados relacionados ao desempenho acadêmico dos alunos em cada método ainda são inconclusivos, pois os valores obtidos nos resultados dos artigos selecionados foram muito semelhantes.

\section{Contribuições das autoras}

Figueiredo TR participou da busca e análise dos dados da pesquisa, interpretação dos resultados, redação e encaminhamento do artigo científico. Aleluia IMB participou da concepção, delineamento, orientação e encaminhamento do artigo científico.

\section{Conflitos de interesses}

Nenhum conflito financeiro, legal ou político envolvendo terceiros (governo, empresas e fundações privadas, etc.) foi declarado para nenhum aspecto do trabalho submetido (incluindo mas não limitando-se a subvenções e financiamentos, conselho consultivo, desenho de estudo, preparação de manuscrito, análise estatística, etc.).

\section{Referências}

1. Krathwohl DR. A Revision of Bloom's Taxonomy: An Overview. Theory Into Practice. 2012;41(4):212-218. doi: 10.1207/ s15430421tip4104_2

2. Bobrowski P. Bloom 's Taxonomy - Expanding its Meaning. Pacific Crest Fac Dev Ser. 2000;(1993):101-4.

3. Interactive EP. Bloom's et al. Taxonomy of the cognitive domain. 2014;(2011):1-4.
4. Veeramani R, Madhugiri VS, Chand P. Perception of MBBS students to "flipped class room" approach in neuroanatomy module. Anat Cell Biol. 2015;48(2):138-43. doi: 10.5115/ $\underline{\text { acb.2015.48.2.138 }}$

5. Sharma N, Lau CS, Doherty I, Harbutt D. How we flipped the medical classroom. Med Teach. 2015;37(4):327-30. doi: 10.3109/0142159X.2014.923821

6. Li S. Flip the classroom. Educ Prim Care. 2015;26(6):438-40. doi: 10.1080/14739879.2015.1101854

7. Moffett J. Twelve tips for "flipping" the classroom. Med Teach. 2015;37(4):331-6. doi: 10.3109/0142159X.2014.943710

8. Rui Z, Lian-rui X, Rong-zheng Y, Jing Z, Xue-hong W, Chuan Z. Friend or Foe? Flipped Classroom for Undergraduate Electrocardiogram Learning: a Randomized Controlled Study. BMC Med Educ. 2017;17(1):53. doi: 10.1186/s12909-017-0881-8

9. Sajid MR, Laheji AF, Abothenain F, Salam Y, AlJayar D, Obeidat A. Can blended learning and the flipped classroom improve student learning and satisfaction in Saudi Arabia? Int J Med Educ. 2016;7:281-5. doi: 10.5116/ijme.57a7.83d4

10. Educause Learning Initiative. 7 Things you should know about flipped classrooms. [Internet]. 2012. Available from: https:// library.educause.edu/-/media/files/library/2012/2/eli7081-pdf.pdf

11. Smith JS. Active learning strategies in the physician assistant classroom - the critical piece to a successful flipped classroom. J Physician Assist Educ. 2014;25(2):46-9.

12. Yarbro J, Arfstrom KM, McKnight K, McKnight P. Flipped learning review 2014. [Internet]. Available from: https:// flippedlearning.org/wp-content/uploads/2016/07/Extension-ofFLipped-Learning-LIt-Review-June-2014.pdf

13. Alexa J, The S, Delta P, September N. 10 reasons to flip. 2014;94(1):41-4.

14. Chen F, Lui AM, Martinelli SM. A systematic review of the effectiveness of flipped classrooms in medical education. Med Educ. 2017;51(6):585-97. doi: 10.1111/medu.13272

15. Morton DA, Colbert-Getz JM. Measuring the impact of the flipped anatomy classroom: The importance of categorizing an assessment by Bloom's taxonomy. Anat Sci Educ. 2017;10(2):1705. doi: $10.1002 /$ ase. 1635

16. Berge ZL. Active, Interactive, and reflective elearning. The Quarterly Review of Distance Education. 2002;3(2):181-190.

17. Freeman S, Eddy SL, Mcdonough M, Smith MK, Okoroafor $\mathrm{N}$, Jordt $\mathrm{H}$ et al. Active learning increases student performance in science, engineering, and mathematics. Proc Natl Acad Sci. 2014.111(23):8410-5. doi: 10.1073/pnas.1319030111 
18. Murray D, Koziniec T, Mcgill T. Student Perceptions of Flipped Learning. In: 17th Australasian Computer Education Conference (ACE 2015). 2015; Sydney, Australia: Conferences in Research and Practice in Information Technology (CRPIT); 2015.

19. Strayer JF. The Effects Of The Classroom Flip On The Learning Environment: A Comparison Of Learning Activity In A Traditional Classroom And A Flip Classroom That Used An Intelligent Tutoring System [dissertation]. Ohio: The Ohio State University; 2007. 